\title{
SPECTRA OF OPERATOR EQUATIONS AND AUTOMORPHISM GROUPS OF VON NEUMANN ALGEBRAS ${ }^{1}$
}

\author{
HERBERT HALPERN
}

\begin{abstract}
.
The spectrum of the operator $T x=\sum \varphi_{i}(a) x \psi_{i}(b)$ in the space of bounded endomorphisms of a von Neumann algebra is computed. Also the spectrum of a locally compact abelian group of automorphisms is computed.
\end{abstract}

\section{Introduction.}

In this article, we compute the spectrum of an operator equation of the form

$$
T x=\sum\left\{\varphi_{i}(a) x \psi_{i}(b) \mid \cdot 1 \leqq i \leqq n\right\}
$$

in the algebra $L(A)$ of bounded linear operators on a von Neumann algebra. Here $a$ and $b$ are elements of $A$ and $\varphi_{i}$ and $\psi_{i}$ complex-valued functions so that $\varphi_{i}(a)$ and $\psi_{i}(b)$ make sense, viz. $\varphi_{i}$ or $\psi_{i}$ is continuous on $\operatorname{Sp} a$ or $\operatorname{Sp} b$ if $a$ or $b$ is normal and $\varphi_{i}$ or $\psi_{i}$ is holomorphic on (domains) containing $\operatorname{Sp} a$ or $\operatorname{Sp} b$ if $a$ or $b$ is not normal. The technique employed also allows us to compute the spectrum of an operator equation of the form (1) when $A$ is a $\mathrm{C}^{*}$-algebra.

Next, we compute the spectrum of a $\sigma$-weakly continuous representation of a locally compact abelian group $G$ as *-automorphisms. Although the spectrum for such an automorphism group is defined differently from the spectrum of an operator equation in that the present spectrum is the intersection of the zero sets of the Fourier transforms of functions of $L^{1}(G)$ in the kernel of the induced representation, we can use the same technique for finding the present spectrum as we used for the operator equation (1) at least when the representation is implemented by a unitary representation of $G$ in $A$. We also characterize the kernel of the representation induced on $L^{1}(G)$ as the set of functions whose Fourier transform vanishes on the spectrum.

1 This research was partially supported by the National Science Foundation.

Received February 7, 1977. 


\section{Operator equations.}

Throughout this article we use the following

Notation. Let $A$ be a von Neumann algebra with center $Z$, let $\Xi$ be the spectrum of $Z$, let $[\xi]$ be the (closed) ideal in $A$ generated by $\xi$ in $\Xi$ and let $a(\xi)$ be the image of $a$ in $A(\xi)=A /[\xi]$ under the canonical homomorphism of $A$ into $A(\xi)$. Let $L(A)$ be the Banach algebra of all bounded linear endomorphisms of $A$.

The space $L_{w}(A)$ of $\sigma$-weakly continuous endomorphisms of $A$ into $A$ is a norm closed subalgebra of $L(A)$. We remark that if $T$ is an operator in $L_{w}(A)$ which is invertible in $L(A)$, then $T^{-1}$ is also in $L_{w}(A)$. In fact, let $A^{\prime \prime}$ be the enveloping von Neumann algebra of $A$ and let $A$ be embedded in $A^{\prime \prime}$. There is a central projection $p$ in $A^{\prime \prime}$ such that $A^{\prime \prime} p=A p$ and $A$ is isomorphic to $A p$. Let $M_{p}$ in $L\left(A^{\prime \prime}\right)$ be given by $M_{p} x=p x$. Now, if $T$ is invertible in $L(A)$, the second transpose $\left(T^{-1}\right)^{t t}$ is the inverse of $T^{t t}$ in $L\left(A^{\prime \prime}\right)$. The operator $M_{p}\left(T^{-1}\right)^{t t}$ maps $A^{\prime \prime} p$ into $A^{\prime \prime} p$ and is $\sigma$-weakly continuous on $A^{\prime \prime} p$. Since $\left(T^{-1}\right)^{t t}$ is the inverse of $T^{t t}$ on $A^{\prime \prime}$, the map $M_{p}\left(T^{-1}\right)^{t t}$ is identified with $T^{-1}$ under the isomorphism of $A$ onto $A^{\prime \prime} p$.

We first compute the spectrum of the operator equation (1) when $\varphi_{i}, \psi_{i}$ are holomorphic. We use the technique of Lumer and Rosenblum [12].

THEOREM 1. Let $a$ and $b$ be elements of $A$, let $\varphi_{i}, \psi_{i}(1 \leqq i \leqq n)$ be holomorphic functions on domains containing $a$ and $b$ respectively; then the. spectrum of the operator equation

$$
T x=\sum \varphi_{i}(a) x \psi_{i}(b)
$$

in the Banach algebra $L(A)$ of all bounded linear operators on the Banach space $A$ is

$$
X=\bigcup_{\xi \in \Xi}\left\{\sum \varphi_{i}(\alpha) \psi_{i}(\beta) \mid \alpha \in \operatorname{Sp} a(\xi), \beta \in \operatorname{Sp} b(\xi)\right\}
$$

Proof. Suppose $\alpha$ is in $\operatorname{Sp} a(\xi)$ and $\alpha-a(\xi)$ does not have a left inverse in $A(\xi)$. There is a projection $e_{n}$ in $A$ with $e_{n}(\xi) \neq 0$ such that

$$
\left\|(\alpha-a) e_{n}(\xi)\right\|<n^{-1} \text {. }
$$

For example, the spectral projection of $(\alpha-a)^{*}(\alpha-a)$ corresponding to the interval $\left[-n^{2}, n^{2}\right]$ suffices. There is a projection $p_{n}$ in $Z$ with $\hat{p_{n}}(\xi)=1$ such that

$$
\left\|(\alpha-a) e_{n} p_{n}\right\|<n^{-1}
$$


due to the continuity of the map $\zeta \rightarrow\|d(\zeta)\|$ on $\Xi$ for fixed $d$ in $A$ [6, Lemma $10]$. By replacing $p_{n}$ by the central support of $e_{n} p_{n}$, we may assume that $p_{n}$ is the central support of $e_{n} p_{n}$. Then the sequence $\left\{e_{n} p_{n}\right\}$ right zero divides $\alpha-a$ in the terminology of Lumer and Rosenblum [12, v]. If $\alpha-a(\xi)$ does not have a right inverse, then there is a sequence $\left\{e_{n} p_{n}\right\}$ of projections in $A$ of central support $p_{n}$ with $\hat{p_{n}}(\xi)=1$ such that $\left\{e_{n} p_{n}\right\}$ left zero divides $\alpha-a$. Now if $\beta$ is in $\operatorname{Sp} b(\xi)$, there is a sequence $\left\{f_{n} q_{n}\right\}$ of projections of central support $q_{n}$ with $\hat{q_{n}}(\xi)=1$ such that $\left\{f_{n} q_{n}\right\}$ either right or left zero divides $\beta-b$. Since $e_{n} p_{n} q_{n}$ and $f_{n} p_{n} q_{n}$ have the same central supports $p_{n} q_{n}$, there is a nonzero partial isometry $u_{n}$ whose domain support is majorized by $f_{n} p_{n} q_{n}$ and whose range support is majorized by $e_{n} p_{n} q_{n}$ [4, III $\S 1$, Lemma 1]. Thus, the number $\sum \varphi_{i}(\alpha) \psi_{i}(\beta)$ is in the spectrum of $T$ [12, Theorem 9].

Now suppose $\gamma$ is not in $X$. Given any $\xi \in \Xi$, we show that there is a projection $p=p_{\xi}$ in $Z$ with $\hat{p}(\xi)=1$ such that $\gamma$ is not in

$$
\left\{\sum \varphi_{i}(\alpha) \psi_{i}(\beta) \mid \alpha \in \mathrm{Sp}_{A p} a p, \beta \in \mathrm{Sp}_{A p} b p\right\} .
$$

On the contrary, suppose for every projection $p$ in $Z$ with $\hat{p}(\xi)=1$ there is an $\alpha_{p}$ in $\operatorname{Sp}_{A p}$ and $\mathrm{a} \beta_{p}$ in $\operatorname{Sp}_{A p} b p$ with $\sum \varphi_{i}\left(\alpha_{p}\right) \psi_{i}\left(\beta_{p}\right)=\gamma$. There are $\zeta_{p}$ and $\xi_{p}$ in $\Xi$ with $\hat{p}\left(\zeta_{p}\right)=\hat{p}\left(\xi_{p}\right)=1$ such that $\alpha_{p}$ and $\beta_{p}$ are in $\operatorname{Sp} a p\left(\zeta_{p}\right)$ and $\operatorname{Sp} b p\left(\xi_{p}\right)$ respectively [15, Lemma 6.2]. The projections $p$ in $Z$ with $\hat{p}(\xi)=1$ form a directed set under the natural ordering for projections in $Z$ and so we may find a subnet $\left\{p_{j}\right\}$ such that

$$
\alpha_{p_{j}}=\alpha_{j} \rightarrow \alpha, \quad \beta_{p_{j}}=\beta_{j} \rightarrow \beta
$$

and

$$
\xi_{p_{j}}=\xi_{j} \rightarrow \xi, \quad \zeta_{p_{j}} \rightarrow \xi .
$$

The element $\alpha$ is in $\operatorname{Sp} a(\xi)$; otherwise, there would be a $c$ in $A$ with

$$
\|(1-c(\alpha-a))(\xi)\|+\|(1-(\alpha-a) c)(\xi)\|=0,
$$

and consequently,

$$
\left\|\left(1-c\left(\alpha_{j}-a\right)\right)\left(\xi_{j}\right)\right\|+\left\|\left(1-\left(\alpha_{j}-a\right) c\right)\left(\xi_{j}\right)\right\|<1
$$

got some $j$ so that $\alpha_{j}-a$ would be invertible. Similarly, we see that $\beta \in \operatorname{Sp} b(\xi)$. This contradicts the choice of $\gamma$ since $\gamma$ is now given by $\gamma=\sum \varphi_{i}(\alpha) \psi_{i}(\beta)$. Therefore, there is a projection $p$ in $Z$ with $\hat{p}(\xi)=1$ such that $\gamma$ is not in

$$
\sum\left\{\varphi_{i}(\alpha) \psi_{i}(\beta) \mid \alpha \in \mathrm{Sp}_{A p} a p, \beta \in \mathrm{Sp}_{A p} b p\right\} .
$$

We have that $\varphi_{i}$ and $\psi_{i}$ are holomorphic on $\mathrm{Sp}_{A p} a p$ and $\mathrm{Sp}_{A p} b p$, respectively and that 


$$
\varphi_{i}(a p)=\varphi_{i}(a) p, \quad \psi_{i}(b p)=\psi_{i}(b) p
$$

due to the Cauchy integral representation for $\varphi_{i}(a)$ and $\psi_{i}(b)$. We may find an operator $S_{p}$ in $L(A p)$ with

$$
S_{p}\left(\gamma-T_{p}\right)=\left(\gamma-T_{p}\right) S_{p}=\text { identity on } L(A p)
$$

where $T_{p}$ is given by

$$
T_{p} x=\sum \varphi_{i}(a p) x \psi_{i}(a p)
$$

for $x$ in $A p$. Setting $M_{p}$ equal to the multiplication operator $M_{p} x=x p$ for $x \in A$, we may rewrite the preceding relation as

$$
M_{p} S_{p} M_{p}(\gamma-T)=(\gamma-T) M_{p} S_{p} M_{p}=M_{p} .
$$

Since there is a finite set $\left\{r_{j}=p_{\xi j} \mid 1 \leqq j \leqq m\right\}$ of projections of least upper bound 1, there is an inverse $S$ for $T$ in $L(A)$ given by $S=\sum M_{q_{j}} S_{r_{j}} M_{q_{j}}$ where $q_{0}=0$ and

$$
q_{j}=\operatorname{lub}\left\{r_{k} \mid 1 \leqq k \leqq j\right\}-\operatorname{lub}\left\{q_{k} \mid 1 \leqq k \leqq j-1\right\}
$$

for $j \geqq 1$. Thus, the complement of $X$ is contained in the complement of $\operatorname{Sp} T$, that is, $\operatorname{Sp} T \subset X$.

We now obtain a more global and also an extended formulation of Theorem 1 when $a$ and $b$ are normal operators. For this the concept of central spectrum is needed (cf. [8]).

Definition 2. An element $c$ in $Z$ is said to be in the central spectrum $Z-\operatorname{Sp} a$ of an element $a$ in $A$ if $\hat{c}(\xi)$ is in $\operatorname{Sp} a(\xi)$ for every $\xi \in \Xi$.

In order to consider normal and self-adjoint operators together we extend a result of P. R. Halmos [7] and also prove a spectral mapping theorem for central spectra. We can also prove similar theorems for essential central spectra (cf. [8]), but we do not need these in the present paper.

LEMMA 3. Let a be a normal operator on a Hilbert space $H$; then there is a selfadjoint operator $b$ in the von Neumann algebra $B$ generated by $a$ and 1 on $H$ and $a$ continuous function $\varphi$ of $\operatorname{Sp} b$ into $\operatorname{Sp} a$ such that $\varphi(b)=a$.

Proof. Setting $\Omega$ equal to the spectrum of $B$, we can find a continuous function $\varphi$ of the Cantor set $S$ in $[0,1]$ onto Sp $a$ and a Borel function $\psi$ of Spa into $S$ such that $\varphi \cdot \psi$ is the identity function on $\operatorname{Spa}$ (cf. [7]). There is a unique $b$ in $B$ such that $\hat{b}(\omega)=\psi(\hat{a}(\omega))$ on an open dense subset $\Omega_{0}$ of $\Omega[10 ; \S 11]$. The range of $\bar{b}$ is contained in $S$ and so $b$ is self-adjoint. Then we have that $\varphi(b)$ 
$=a$ because

$$
\varphi(b) \hat{(}(\omega)=\hat{\varphi(b)}(\omega))=\varphi \cdot \psi(\hat{a}(\omega))=\hat{a}(\omega)
$$

for all $\omega$ in $\Omega_{0}$.

The relation between the spectrum and the central spectrum is given in the next proposition.

Proposition 4. Let a be a normal operator in $A$; then $\operatorname{Sp} a(\xi)$ is equal to $\{\hat{c}(\xi) \mid c \in Z-\operatorname{Sp} a\}$.

Proof. First assume $a$ is self-adjoint. There is no loss of generality in assuming $a \geqq 1$. We show that

$$
S(\xi)=\{\hat{c}(\xi) \mid a \in Z-\operatorname{Sp} a\}
$$

is a closed set. It is sufficient to consider the case of a sequence $\left\{c_{n}\right\}$ in $Z-\operatorname{Sp} a$ with $\lim \hat{c_{n}}(\xi)=\alpha$ and $\hat{c_{n}}(\xi)<\alpha$. There is a projection $p$ in $Z$ with $\hat{p}(\xi)=1$ such that $c_{1} p \leqq \alpha p$. The set

$$
S=\{c \in Z-\operatorname{Sp} a \mid \dot{c p} \leqq \alpha p\}
$$

has a least upper bound $c_{0}$ since it is monotonely increasing and strongly closed [7, 3.9 and 3.10]. Then we must have $\hat{c_{0}}(\xi)=\alpha$ and so $S(\xi)$ is closed.

We now show that an arbitrary $\beta$ in $\operatorname{Sp} a(\xi)$ is contained in $S(\xi)$. In fact, let $e(\lambda)$ be the spectral resolution of $a$ and let $e=e(\beta+\varepsilon)-e(\beta-\varepsilon)$ for $0<\varepsilon<\frac{1}{2}$. We have that $e(\xi) \neq 0$; otherwise, we have

$$
(\beta-a)(\xi)=(\beta-a(1-e))(\xi)
$$

is invertible in $A(\xi)$. So we have

$$
\|a e(\beta+\varepsilon)(\xi)\| \geqq(\beta-\varepsilon)\|e(\xi)\|=\beta-\varepsilon .
$$

There is a $c$ in $Z-\operatorname{Sp} a e(\beta+\varepsilon)$ with $2^{-1} \leqq \beta+\varepsilon \leqq \mathrm{c}^{\hat{c}}(\xi) \leqq \beta+\varepsilon[7,3.12]$. Arguing by contradiction, we can show that $\hat{c}(\zeta)$ is in $\operatorname{Spa}(\zeta)$ whenever $\zeta$ is in the nonvoid open subset of $\zeta \in \Xi$ with $\hat{c}(\zeta)>0$ and so $\hat{c}(\xi)$ is in $S(\xi)[7,3.10]$. Since $S(\xi)$ is closed, we get that $\beta \in S(\xi)$. Hence, we have that $\operatorname{Sp} a(\xi)$ is contained in $S(\xi)$. Because the reverse inclusion relation follows from Definition 2, we have that $S(\xi)=\operatorname{Sp} a(\xi)$.

Now let $a$ be a normal operator in $A$. There is a self-adjoint operator $b$ in $A$ and a continuous complex-valued function $\varphi$ on $\operatorname{Sp} a$ with $\varphi(b)=a$ [Lemma 3]. Notice that $\varphi(c)$ is defined for every $c$ in $Z-\operatorname{Sp} b$, and that $\{\varphi(c) \mid c \in Z-\operatorname{Sp} b\}$ is contained in $Z-\mathrm{Sp} a$. Thus, we get that 


$$
\begin{aligned}
\operatorname{Sp} a(\xi)=\varphi(\operatorname{Sp} b(\xi)) & =\{\varphi(\hat{c}(\xi)) \mid c \in Z-\operatorname{Sp} b\} \\
& =\{\hat{c}(\xi) \mid c \in Z-\operatorname{Sp} a\}
\end{aligned}
$$

from the first part of the proof.

Lemma 5. Let a be a normal operator in $A$ and let $\varphi$ be a continuous complexvalued function on the spectrum of $a$; then $\varphi(Z-\operatorname{Sp} a)=Z-\operatorname{Sp} \varphi(a)$.

Proof. Let $b$ be a self-adjoint operator in $A$ and let $\psi$ be a continuous complex-valued function on $\operatorname{Sp} b$ such that $\psi(b)=a$ (Lemma 3). Let $\theta=\varphi \cdot \psi$. Given $c$ in $Z-\operatorname{Sp} b$ and $\xi$ in $\Xi$, there is a $d_{\xi}$ in $Z-\operatorname{Sp} b$ with $\theta\left(d_{\xi}\right) \hat{(\xi)}=\hat{c}(\xi)$ due to the classical Spectral Mapping Theorem and Lemma 4. Using the fact that $\Xi$ is extremally disconnect, we can find a sequence $\left\{d_{n}\right\}$ in $Z-\operatorname{Sp} b$ with $\left\|\theta\left(d_{n}\right)-c\right\| \leqq n^{-1}$ for $n=1,2, \ldots$. The operators

$$
d_{n k}=\operatorname{lub}\left\{d_{n}, d_{n+1}, \ldots, d_{n+k}\right\}
$$

are in $Z-\operatorname{Sp} b[7,3.10]$ and satisfy the relation

$$
\left\|\theta\left(d_{n k}\right)-c\right\| \leqq n^{-1}
$$

for every $k \geqq 0$. We have that $d=\operatorname{glb}_{n}\left(\operatorname{lub}_{k} d_{n k}\right)$ is in the strongly closed set $Z$ $-\operatorname{Sp} b$ and that $\theta(d)=c$. Thus we have that $c$ in $Z-\operatorname{Sp} \varphi(a)$ is the image of $\psi(d)$ in $Z-\operatorname{Sp} a$ under $\varphi$. This proves that $Z-\operatorname{Sp} \varphi(a)$ is contained in $\varphi(Z-\operatorname{Sp} a)$. The opposite containment relation is clear and thus $\varphi(Z-\operatorname{Sp} a)=Z-\operatorname{Sp} \varphi(a)$.

We now can prove the extended global formulation of Theorem 1.

THEOREM 6. Let $a$ and $b$ be normal operators in $A$, let $\varphi_{i}$ and $\psi_{i}(1 \leqq i \leqq n)$ be continuous functions on $\mathrm{Sp} a$ and $\mathrm{Sp} b$ respectively; then the spectrum of the operator $T$ in $L(A)$ defined by

$$
T x=\sum \varphi_{i}(a) x \psi_{i}(b)
$$

is given by

$$
\begin{aligned}
X & =\bigcup_{\xi \in \Xi}\left\{\sum \varphi_{i}(\alpha) \psi_{i}(\beta) \mid \alpha \in \operatorname{Sp} a(\xi), \beta \in \operatorname{Sp} b(\xi)\right\} \\
& =\bigcup_{\xi \in \Xi}\left\{\left(\sum \varphi_{i}(c) \psi_{i}(d)\right)^{\hat{\gamma}}(\xi) \mid c \in Z-\operatorname{Sp} a, d \in Z-\operatorname{Sp} b\right\}
\end{aligned}
$$

Proof. First we notice that the equivalence of the two expressions for $X$ is obtained from Lemma 5. There is no loss of generality in assuming that both $a$ and $b$ are self-adjoint (Lemma 3). There are sequences $\left\{\varphi_{i j}\right\}_{j}$ and $\left\{\psi_{i j}\right\}_{j}$ of 
holomorphic functions on $\operatorname{Sp} a$ and $\operatorname{Sp} b$ that converge uniformly to $\varphi_{i}$ and $\psi_{i}$ respectively. Setting

$$
T_{j} x=\sum_{i} \varphi_{i j}(a) x \psi_{i j}(b) \quad \text { for } j=1,2, \ldots,
$$

we obtain a sequence $\left\{T_{j}\right\}$ of mutually commuting operators in $L(A)$ converging to $T$. The sequence $\left\{\sum_{i} \varphi_{i j}(\alpha) \psi_{i j}(\beta)\right\}$ converges to $\sum \varphi_{i}(\alpha) \psi_{i}(\beta)$ for every $\alpha$ in $\operatorname{Sp} a(\xi)$ and $\beta$ in $\operatorname{Sp} b(\xi)$.

Now let $\gamma$ be an element in $X$ given by $\gamma=\sum \varphi_{i}(\alpha) \psi_{i}(\beta)$ with $\alpha$ in Sp $a(\xi), \beta$ in $\operatorname{Sp} b(\xi)$. We obtain a contradiction from the assumption that $\gamma$ is not in $\operatorname{Sp} T$. There is an $S$ in $L(A)$ with

$$
\|1-S(\gamma-T)\|+\|1-(\gamma-T) S\|=0 .
$$

Setting $\gamma_{j}=\sum_{i} \varphi_{i j}(\alpha) \psi_{i j}(\beta)$ for $j=1,2, \ldots$, we may find a $j$ such that

$$
\left\|1-S\left(\gamma_{j}-T_{j}\right)\right\|+\left\|1-\left(\gamma_{j}-T_{j}\right) S\right\|<1 .
$$

This means $\gamma_{j}-T_{j}$ is invertible. This is impossible (Theorem 1). Hence, we have that $X$ is contained in $\operatorname{Sp} T$.

Conversely, assume that' $\gamma$ is not in $X$. We notice that $X$ is a closed set. In fact, let $\left\{\delta_{n}\right\}$ be a sequence in $X$ with $\lim \delta_{n}=\delta$, and let $\alpha_{n}$ and $\beta_{n}$ be in $\operatorname{Sp} a\left(\xi_{n}\right)$ and $\operatorname{Sp} b\left(\xi_{n}\right)$ respectively with $\delta_{n}=\sum \varphi_{i}\left(\alpha_{n}\right) \psi_{i}\left(\beta_{n}\right)$. By passing to subnets, we may assume that $\left(\alpha_{n}, \beta_{n}, \xi_{n}\right)$ converges to $(\alpha, \beta, \xi)$ in $C \times C \times \Xi$. We have that $\alpha \in \operatorname{Sp} a(\xi), \beta \in \operatorname{Sp} b(\xi)$ (as we have already shown), and

$$
\delta=\lim _{n} \sum \varphi_{i}\left(\alpha_{n}\right) \psi_{i}\left(\beta_{n}\right)=\sum \varphi_{i}(\alpha) \psi_{i}(\beta)
$$

is in $X$; and consequently, we get that $X$ is closed. Now there is a neighborhood $V$ of 0 in the complex plane so that $\gamma$ is not in $X+2 V$. Also there is a $j_{0}$ such that $j \geqq j_{0}$ implies

$$
X_{j}=\bigcup_{\xi \in \Xi}\left\{\sum \varphi_{i j}(\alpha) \psi_{i j}(\beta) \mid \alpha \in \operatorname{Sp} a(\xi), \beta \in \operatorname{Sp} b(\xi)\right\}
$$

is contained in $X+V$. Thus $\gamma$ is not in $\operatorname{Sp} T_{j}+V$ for every $j \geqq j_{0}$ (Theorem 1 ). This means that $\gamma$ is not in $\operatorname{Sp} T$. Indeed, there is an $\varepsilon>0$ such that $\left\|T-T_{j}\right\|<\varepsilon$ implies $\operatorname{Sp} T \subset \operatorname{Sp} T_{j}+V[13,1.6 .17]$. So we have that $\operatorname{Sp} T \subset X$.

Now we can characterize the spectra of operator equations of the form (1) on $\mathrm{C}^{*}$-algebras.

Proposition 7. Let $B$ be a $\mathrm{C}^{*}$-algebra, let $B^{\prime \prime}$ be the enveloping von Neumann algebra of $B$, let a (respectively $b$ ) be an element of $B^{\prime \prime}$ and let $\varphi_{i}$ (respectively $\psi_{i}$ ) $(1 \leqq i \leqq n)$ be holomorphic functions on $\operatorname{Sp} a$ (respectively $\mathrm{Sp} b$ ) or continuous 
complex-valued functions on $\operatorname{Spa}$ (respectively $\mathrm{Sp} b$ ) if a (respectively b) is $a$ normal operator. Suppose the operator $T x=\sum \varphi_{i}(a) x \psi_{i}(b)$ on $B^{\prime \prime}$ maps $B$ into $B$. Then the spectrum of $S=T \mid B$ in $L(B)$ is equal to the spectrum of $T$ in $L\left(B^{\prime \prime}\right)$.

Proof. First let $\alpha \in \operatorname{Sp} S$. We may assume there is an $\varepsilon>0$ such that $\|(\alpha-S) x\| \geqq \varepsilon\|x\|$ for every $x$ in $B$; otherwise, there is a sequence $\left\{x_{n}\right\}$ of elements of unit norm in $B$ with $\lim (\alpha-S) x_{n}=0$ and so $\alpha$ is certainly in $\operatorname{Sp} T$. There is a nonzero continuous linear functional $\omega$ on $B$ which vanishes on $(\alpha-S)(B)$ since $(\alpha-S)(B)$ must be a proper closed subspace of $B[9,2,12.1]$. Let $\omega^{\prime}$ be the unique extension of $\omega$ to a $\sigma$-weakly continuous linear functional on $B^{\prime \prime}$. Because $\omega^{\prime}\left((\alpha-T) B^{\prime \prime}\right)$ is contained in the closure of $\omega((\alpha-S)(B))$, we see that the range of $(\alpha-T)\left(B^{\prime \prime}\right)$ is a proper subset of $B^{\prime \prime}$. So we get $\alpha-T$ is not invertible in $L\left(B^{\prime \prime}\right)$.

Conversely, suppose that $\alpha$ is not in $\operatorname{Sp} S$. Then the double transposes $\left((\alpha-S)^{-1}\right)^{t t}$ and $(\alpha-S)^{t t}$ are $i$-weakly continuous maps of $L\left(B^{\prime \prime}\right)$. Here $B^{\prime \prime}$ is identified with the second dual of $B$. Since $\left((\alpha-S)^{-1}\right)^{t t}$ is the inverse of $(\alpha-S)^{t t}=\alpha-T$, we get that $\alpha$ is not in $\operatorname{Sp} T$.

Corollary 8. Let $B$ be a $C^{*}$-algebra on the Hilbert space $H$, let $A$ be the von Neumann algebra generated by $B$, let a (respectively $b$ ) be an element of $A$, let $\varphi_{i}$ (respectively $\left.\psi_{i}\right)(1 \leqq i \leqq n)$ be holomorphic functions on $\mathrm{Sp} a$ (respectively $\mathrm{Sp} b$ ) or continuous complex-valued functions on $\mathrm{Sp} a$ (respectively $\mathrm{Sp} b$ ) if a (respectively $b$ ) is normal. Suppose $T x=\sum \varphi_{i}(a) x \psi_{i}(b)$ on $A$ maps $B$ into $B$ and that

$$
X=\bigcup_{\xi \in \Xi}\left\{\sum \varphi_{i}(\alpha) \psi_{i}(\beta) \mid \alpha \in \operatorname{Sp} a(\xi), \beta \in \operatorname{Sp} b(\xi)\right\}
$$

fails to separate the plane. There the spectrum of $S=T \mid B$ in $L(B)$ is $X$.

Proof. Let $L_{B}$ be the Banach subalgebra of all operators in $L(A)$ which map $B$ into $B$. Then $T$ is in $L_{B}$ and $\operatorname{Sp} T=X$ is equal to the spectrum of $T$ in $L_{B}$ [16, 1.6.13]. The latter is just the spectrum of $S$ in $L(B)$.

In general, one always has $\operatorname{Sp} T \subset \operatorname{Sp} S$.

EXAMPLE 9. Let $\delta$ be a derivation of the $C^{*}$-algebra $B$ with $\delta^{*}=-\delta$, that is, $\delta\left(b^{*}\right)^{*}=-\delta(b)$ for all $b \in B$. Let $\Gamma$ be a family of representations $\pi$ on the Hilbert spaces $H_{\pi}$ such that the direct sum $\pi_{0}$ of the representations acting on the direct sum $H_{0}$ of the Hilbert spaces is faithful. There is an $a=a^{*}$ in the von Neumann algebra $A$ generated by $\pi_{0}(B)$ such that $\pi_{0}(\delta(x))=\pi_{0}(x) a-a \pi_{0}(x)$ for all $x \in B$ [14]. Now the spectrum of the derivation ad $a$ on $A$ given by ad $a(x)$ $=x a-a x$ is a subset of the real numbers (Theorem 1) and so Sp ad $a$ is equal to 
$\operatorname{Sp} \delta$. In particular, if $\Gamma$ is a separating family of irreducible (respectively factor) representations of $B$, we get

$$
\begin{aligned}
\operatorname{Sp} \delta & =\bigcup_{\xi \in \Xi}\left\{\alpha_{1}-\alpha_{2} \mid \alpha_{i} \in \operatorname{Sp} a(\xi)\right\} \\
& =\bigcup_{\xi \in \Xi}\left\{\left(c_{1}-c_{2}\right) \hat{(\xi)} \mid c_{i} \in Z-\operatorname{Sp} a\right\} \\
& =\operatorname{clos} \bigcup\left\{\left(c_{1}-c_{2}\right) \hat{(\xi)} \mid\{\xi\} \text { open in } \Xi, c_{i} \in Z-\operatorname{Sp} a\right\} \\
& =\operatorname{clos} \bigcup_{\pi \in \Gamma}\left\{\alpha_{1}-\alpha_{2} \mid \alpha_{i} \in \operatorname{Sp} a_{\pi}\right\},
\end{aligned}
$$

where $a_{\pi}$ is any self-adjoint operator in the von Neumann algebra generated by $\pi(B)$ on $H_{\pi}$ with $\pi(\delta(b))=\pi(b) a_{\pi}-a_{\pi} \pi(b)$. The last result is mentioned by C. Akemann and P. Ostrand [1].

\section{Spectra of automorphism groups.}

Let $G$ be a locally compact abelian group and let $A$ be a von Neumann algebra with center $Z$ on the Hilbert space $H$. A homomorphism $\sigma$ of $G$ into the group Aut $A$ of *-automorphisms of $A$ such that $t \rightarrow \omega\left(\sigma_{t}(x)\right)$ is continuous for each $\omega$ in the set $A_{*}$ of $\sigma$-weakly continuous functionals of $A$ is called a representation of $G$ on $A$. If $\sigma$ is a representation of $G$ on $A$ and if $\varphi$ is in $L^{1}(G)$, the relation

$$
\omega(\sigma(\varphi) x)=\int \varphi(t) \omega\left(\sigma_{t}(x)\right) d t \quad\left(x \in A, \omega \in A_{*}\right)
$$

determines an element $\sigma(\varphi)$ in the Banach algebra $L_{w}(A)$ of $\sigma$-weakly continuous linear endomorphisms of $A$. The spectrum $\operatorname{Sp} \sigma$ of $\sigma$ is defined to be the set of all $\chi$ in the dual group $\hat{G}$ of $G$ such that the Fourier transform $\hat{\varphi}$ of $\varphi$ vanishes at $\chi$ whenever $\sigma(\varphi)$ vanishes [2]. The representation $\sigma$ is said to be unitarily implemented by the strongly continuous unitary representation $u$ of $G$ on $H$ if $\sigma_{t}(x)=u_{t} x u_{t}^{*}$ for all $t \in G$ and $x \in A$. If $\sigma$ is unitarily implemented by $u$, there is a projection-valued measure $e$ on the Baire sets $B(\hat{G})$ of the dual group $\hat{G}$ of $G$ (i.e., a map $e$ of the $\sigma$-ring of $\hat{G}$ generated by the compact $G_{\delta}$-sets of $\hat{G}$ into the projections on $H$ such that $e(\varnothing)=0$, $e\left(X_{1} \cap X_{2}\right)=e\left(X_{1}\right) e\left(X_{2}\right)$ for $X_{1}, X_{2}$ in $B(\hat{G})$, and $e\left(\cup X_{i}\right)=\sum e\left(X_{i}\right)$ for a mutually disjoint sequence $\left\{X_{i}\right\}$ in $B(\hat{G}))$ such that

$$
\left(u_{t} \eta, \varrho\right)=\int\langle t, \chi\rangle^{-} d(e(\chi) \eta, \varrho)
$$

for all $\eta, \varrho$ in $H$ (Stone's Theorem, cf. $[11,36])$. Here $\langle t, \chi\rangle$ denotes the action of $\hat{G}$ on $G$. If $e$ can be chosen to be in $A$ (i.e. with range in $A$ ), then $\sigma$ is said to be an inner representation of $G$ on $A$.

Now let $e$ be a projection-valued measure in $A$. 
Definition 10. For every $\xi$ in the spectrum $\Xi$ of $Z$, the spectrum $\operatorname{Sp} e(\xi)$ of $e(\xi)$ is defined to be the set of all $\chi \in \hat{G}$ such that $e(\chi+V)(\xi) \neq 0$ for every $V$ in the family $V(\hat{G})$ of neighborhoods of the identity in $B(\hat{G})$.

We now characterize the spectrum and kernel of an inner representation. D. Olesen [12] has given a similar characterization for the spectrum of an inner automorphism group on the algebra of all bounded operators on a Hilbert space and A. Connes [2] has shown that a function is in the kernel of the induced representation of $L^{1}(G)$ if its Fourier transform vanishes in a neighborhod of the spectrum.

THEOREM 11. Let $\sigma$ be an inner representation of the locally compact abelian group $G$ on the von Neumann algebra $A$ and let e be a projection-valued measure on $B(\hat{G})$ in $A$ such that the unitary representation of $G$ given by $u_{t}$ $=\int\langle t, \chi\rangle^{-} \operatorname{de}(\chi)$ implements $\sigma$; then the spectrum of $\sigma$ is given by

$$
\operatorname{Sp} \sigma=\operatorname{clos}\left\{\chi_{1}-\chi_{2} \mid \chi_{i} \in \operatorname{Spe}(\xi), \xi \in \Xi\right\}
$$

and the kernel of $\sigma$ is given by

$$
\operatorname{ker} \sigma=\left\{\varphi \in L^{1}(G) \mid \hat{\varphi}(\operatorname{Sp} \sigma)=\{0\}\right\} .
$$

Proof. Let $\varphi$ be a function in the kernel of $\sigma$, let $\xi \in \Xi$, and let $\chi_{1}, \chi_{2}$ be in Spe $(\xi)$. From the relations

$$
\int \varphi(t) \sigma_{t}\left(e\left(\chi_{1}+V\right) x e\left(\chi_{2}+V\right)\right) d t=\sigma(\varphi)\left(e\left(\chi_{1}+V\right) x e\left(\chi_{2}+V\right)\right)=0
$$

and

$$
\begin{aligned}
& \left\|\sigma_{t}\left(e\left(\chi_{1}+V\right) x e\left(\chi_{2}+V\right)\right)-\left\langle t, \chi_{2}\right\rangle\left\langle t, \chi_{1}\right\rangle^{-} e\left(\chi_{1}+V\right) x e\left(\chi_{2}+V\right)\right\| \\
& \quad \leqq 2\|x\| \operatorname{lub}\left\{\sum\left|\left\langle t, \chi_{i}+\chi_{i}^{\prime}\right\rangle-\left\langle t, \chi_{i}\right\rangle\right| \chi_{i}^{\prime} \in V, i=1,2\right\} \\
& \quad \leqq\|x\| \chi_{V}(t)
\end{aligned}
$$

we get

$$
\|\left(\int \varphi(s)\left\langle s, \chi_{1}-\chi_{2}\right\rangle^{-} d s\right)\left(e\left(\chi_{1}+V\right) x e\left(\chi_{2}+V\right)\|\leqq\| x\|\| \varphi \chi_{V} \|_{1} .\right.
$$

Since there is a partial isometry $w$ in $A$ with $\left\|e\left(\chi_{1}+V\right) w e\left(\chi_{2}+V\right)\right\|=1[4$, III,1, Lemma 1] and since $\left\|\varphi x_{V}\right\|_{1}$ approaches 0 as $V$ gets small, we conclude $\hat{\varphi}\left(\chi_{1}-\chi_{2}\right)=0$.

Conversely, let $\hat{\varphi}\left(\chi_{1}-\chi_{2}\right)=0$ for all $\chi_{1}, \chi_{2}$ in $\operatorname{Sp} e(\xi)(\xi \in \Xi)$. Let $\eta$ and $\varrho$ be in $H$, let $\left\{X_{i}\right\}$ be a monotonely increasing sequence of compact sets in $B(\hat{G})$ with 


$$
\lim e\left(X_{i}\right) \eta=\eta \quad \text { and } \quad \lim e\left(X_{i}\right) \varrho=\varrho
$$

(cf. [11]), and let $0<\varepsilon<1$. Let $G_{0}$ be a compact set in $G$ with complement $F$ such that $\int_{F}|\varphi| d t<\varepsilon$. For each $X_{i}$, there is a compact covering $\left\{X_{i j} \mid 1 \leqq i \leqq n_{i}\right\}$ of $X_{i}$ in $B(\hat{G})$ such that

$$
\left\|\langle t, \chi\rangle^{-} e\left(X_{i j}\right)-u_{t} e\left(X_{i j}\right)\right\|<\varepsilon
$$

for every $t \in G_{0}$ and $\chi$ in $X_{i j}$. Let $p_{i j}$ be the central support of $e\left(X_{i j}\right)$ and let $\Xi_{i j}$ be the support of $\hat{p_{i j}}$ in $\Xi$. We now show that there is a continuous map $\lambda_{i j}$ of $\Xi_{i j}$ to $X_{i j}$ such that $\lambda_{i j}(\xi)$ is in $\operatorname{Sp} e(\xi)$ for all $\xi$ in $\Xi_{i j}$. Let $\Pi_{i j}$ be the set

$$
\Pi_{i j}=\left\{(\xi, \chi) \in \Xi_{i j} \times X_{i j} \mid \chi \in \operatorname{Sp} e(\xi)\right\} .
$$

In order to show the existence of such a map $\lambda_{i j}$ it is sufficient to show that $\Pi_{i j}$ is a closed subset of $\Xi_{i j} \times X_{i j}$ and that the map of $\Pi_{i j}$ onto its first coordinate is a surjection onto $\Xi_{i j}$ [5] (cf. [16]). For the first, let $\left\{\left(\xi_{n}, \chi_{n}\right)\right\}$ be a net in $\Pi_{i j}$ that converges to $(\xi, \chi)$ in the compact set $\Xi_{i j} \times X_{i j}$. If $(\xi, \chi)$ is not in $\Pi_{i j}$ there would be a $V$ in $V(\hat{G})$ with $e(\chi+V)(\xi)=0$. Since the two-valued map $\zeta \rightarrow\|e(\chi+V)(\zeta)\|$ is continuous [6], there would be a $U \in V(\hat{G})$ with $\left\|e\left(\chi_{n}+U\right)\left(\xi_{n}\right)\right\|=0$ for some $n$. This is not possible. So $\Pi_{i j}$ must be a closed set. For the second, let $\xi \in \Xi_{i j}$. If there is no $\chi \in X_{i j}$ with $\chi \in \operatorname{Sp} e(\xi)$, then, for every $\chi$ in $X_{i j}$, there would be a $V_{\chi}$ in $V(\hat{G})$ with $e\left(\chi+V_{\chi}\right)(\xi)=0$. Since $X_{i j}$ is compact, there would be a finite subset of $\left\{\chi+V_{\chi} \mid \chi \in X_{i j}\right\}$ which would cover $X_{i j}$ and so $e\left(X_{i j}\right)(\xi)$ would be zero. This also is not possible. So there is a $\chi$ in $X_{i j}$ such that $(\xi, \chi)$ is in $\Pi_{i j}$. Thus, the map $\lambda_{i j}$ exists.

For each $t$ in $G$, the unitary operator $\left\langle t, \lambda_{i j}\right\rangle$ in $Z_{p_{i j}}$ given by

$$
\left\langle t, \lambda_{i j}\right\rangle \hat{(}(\xi)=\left\langle t, \lambda_{i j}(\xi)\right\rangle
$$

for $\xi$ in $\Xi_{i j}$ satisfies the relation

$$
\begin{aligned}
& \left\|\left\langle t, \lambda_{i j}\right\rangle^{*} e\left(X_{i j}\right)-u_{t} e\left(X_{i j}\right)\right\| \\
& \quad=\operatorname{lub}\left\{\left\|\left(\left\langle t, \lambda_{i j}\right\rangle^{*} e\left(X_{i j}\right)-u_{t} e\left(X_{i j}\right)\right)(\xi)\right\| \mid \xi \in \Xi_{i j}\right\} \\
& \quad \leqq \operatorname{lub}\left\{\left\|\left\langle t, \lambda_{i j}(\xi)\right\rangle^{-} e\left(X_{i j}\right)-u_{t} e\left(X_{i j}\right)\right\| \mid \xi \in \Xi_{i j}\right\} \\
& \quad \leqq \varepsilon
\end{aligned}
$$

Setting $\left\{Y_{i j} \mid 1 \leqq j \leqq n\right\}$ equal to a partition of $X_{i}$ in $B(\hat{G})$ with $Y_{i j} \subset X_{i j}$, we have that

$$
\left\|\sum_{j}\left\langle t, \hat{\lambda}_{i j}\right\rangle^{*} e\left(Y_{i j}\right)-u_{t} e\left(X_{i}\right)\right\| \leqq \operatorname{lub}\left\|\left\langle t, \lambda_{i j}\right\rangle^{*} e\left(Y_{i j}\right)-u_{t} e\left(Y_{i j}\right)\right\|<\varepsilon,
$$


and consequently, that

$$
\begin{array}{r}
\left\|\int \varphi(t) \sigma_{t}\left(e\left(X_{i}\right) x e\left(X_{i}\right)\right) d t-\int \sum_{j, k} \varphi(t)\left\langle t, \lambda_{i j}\right\rangle\left\langle t, \lambda_{i k}\right\rangle^{*} e\left(Y_{i k}\right) x e\left(Y_{i j}\right) d t\right\| \\
\leqq 3 \varepsilon\|x\|\left(1+\|\varphi\|_{1}\right) .
\end{array}
$$

By approximating $\varphi$ in the norm of $L^{1}(G)$ by continuous functions of compact support, we obtain

$$
\left(\int \varphi(t)\left\langle t, \lambda_{i j}\right\rangle\left\langle t, \lambda_{i k}\right\rangle^{*} d t\right) \hat{(\xi)}=\hat{\varphi}\left(\lambda_{i k}(\xi)-\lambda_{i j}(\xi)\right)=0
$$

for $\xi$ in $\Xi_{i j} \cap \Xi_{i k}$. This means that

$$
\left\|\int \varphi(t) \sigma_{t}\left(e\left(X_{i}\right) x e\left(X_{i}\right)\right) d t\right\| \leqq 3 \varepsilon\|x\|\left(1+\|\varphi\|_{1}\right) .
$$

Because $\sigma_{t}\left(e\left(X_{i}\right)\right)=e\left(X_{i}\right)$ for all $t$ and $i$, we have that

$$
\begin{aligned}
\left|\left(\int \varphi(t) \sigma_{t}(x) d t \eta, \varrho\right)\right| & =\left|\int \varphi(t)\left(\sigma_{t}(x) \eta, \varrho\right) d t\right| \\
& =\lim \left|\int \varphi(t)\left(\sigma_{t}\left(e\left(X_{i}\right) x e\left(X_{i}\right)\right) \eta, \varrho\right) d t\right| \\
& \leqq 3 \varepsilon\|x\|\left(1+\|\varphi\|_{1}\right)\|\eta\|\|\varrho\|
\end{aligned}
$$

by the Dominated Convergence Theorem. Since $\varepsilon, \eta, \varrho$ are arbitrary, we get $\sigma(\varphi)(x)=0$. Thus, we get that $\sigma(\varphi)=0$ if and only if $\hat{\varphi}(\chi)=0$ for all $\chi$ in $\left\{\chi_{1}-\chi_{2} \mid \chi_{i} \in \operatorname{Sp} e(\xi), \xi \in \Xi\right\}$. Thus, we have proved that

$$
\operatorname{Sp} \sigma=\operatorname{clos}\left\{\chi_{1}-\chi_{2} \mid \chi_{i} \in \operatorname{Sp} e(\xi), \xi \in \Xi\right\}
$$

and

$$
\operatorname{ker} \sigma=\left\{\varphi \in L^{1}(G) \mid \hat{\varphi}(\operatorname{Sp} \sigma)=\{0\}\right\}
$$

One may give a global formulation of Theorem 11 analogous to the global formulation of Theorem 1 given in Theorem 6. For this let $e$ be a projectionvalued measure in $A$ on $B(\hat{G})$. A continuous function $\lambda$ with maximal graph from an open dense subset $D(\lambda)$ of $\Xi$ into $\hat{G}$ is said to be in the central spectrum $Z$-Spe of $e$ if $\lambda(\xi) \in$ Sp $e(\xi)$ for all $\xi \in D(\lambda)$. If $\lambda(D(\lambda))$ is bounded, then $D(\lambda)$ must be equal to $\Xi$ due to [5]. For example, if $e$ is the spectral resolution of a bounded self-adjoint operator $a$ in $A$ (in which case $\hat{G}$ is the additive group of real numbers), then the $Z-\operatorname{Sp} a$ is identified with $Z-\operatorname{Sp} e$ under the map $c \rightarrow \hat{c}$. This provides a link between the material in sections 2 and 3 . Then 
preserving the notation of Theorem 11, we can reformulate these results as follows: the spectrum of $\sigma$ is given by

$$
\operatorname{Sp} \sigma=\operatorname{clos}\left\{\lambda_{1}(\xi)-\lambda_{2}(\xi) \mid \xi \in D\left(\lambda_{1}\right) \cap D\left(\lambda_{2}\right), \lambda \in Z-\operatorname{Sp} e\right\}
$$

and

$$
\operatorname{ker} \sigma=\left\{\varphi \in L^{1}(G) \mid \hat{\varphi} \cdot\left(\lambda_{1}-\lambda_{2}\right)=0, \lambda_{i} \in Z-\operatorname{Sp} e\right\} .
$$

Here $\hat{\varphi} \cdot\left(\lambda_{1}-\lambda_{2}\right)$ is the unique extension of $\xi \rightarrow \hat{\varphi}\left(\lambda_{1}(\xi)-\lambda_{2}(\xi)\right)$ to a continuous function on $Z$.

We now characterize the kernel of an arbitrary representation $\sigma$ of the locally compact abelian group $G$ on $A$. For this we need the notion of a crossed product. Let $L^{2}(H ; G)$ be the completion of the preHilbert space of all continuous functions of $G$ into $H$ with compact support and inner product

$$
\langle\eta, \varrho\rangle=\int(\eta(t), \varrho(t)) d t .
$$

There is a faithful normal representation $\pi_{\sigma}$ of $A$ onto $L^{2}(G ; H)$ given by

$$
\left(\pi_{\sigma}(x) \eta\right)(t)=\sigma_{t}^{-1}(x) \eta(t)
$$

and there is a strongly continuous unitary representation $u=u_{\sigma}$ of $G$ on $L^{2}(H ; G)$ given by

$$
(u(t) \eta)(s)=\eta(s-t) .
$$

The von Neumann algebra on $L^{2}(H ; G)$ generated by $\pi_{\sigma}(A)$ and the image of $G$ under $u$ is called the crossed product of $A$ by the action of $\sigma$ on $G$ and is denoted by $R(A ; \sigma)$. For $x \in A, t$ in $G$, the relation

$$
u(t) \pi_{\sigma}(x) u(t)^{*}=\pi_{\sigma}\left(\sigma_{t}(x)\right)
$$

holds.

THEOREM 12. Let $\sigma$ be a representation of the locally compact abelian group $G$ on the von Neumann algebra $A$. Then the spectrum of $\sigma$ is equal to the spectrum of the representation $\tilde{\sigma}$ of $G$ on the crossed product $R(A ; \sigma)$ given by $\tilde{\sigma}_{t}(x)$ $=u_{\sigma}(t) x u_{\sigma}(t)^{*}$, and the kernel of the representation of $\sigma$ of $L^{1}(G)$ is given by

$$
\operatorname{ker} \sigma=\left\{\varphi \in L^{1}(G) \mid \hat{\varphi}(\operatorname{Sp} \sigma)=\{0\}\right\} \text {. }
$$

Proof. Let $\varphi$ be in $L^{1}(G)$. We show $\sigma(\varphi)=0$ if and only if $\tilde{\sigma}(\varphi)=0$. On the one hand, we have 


$$
\begin{aligned}
\tilde{\sigma}(\varphi) \pi_{\sigma}(x) & =\int \varphi(t) \tilde{\sigma}_{t}\left(\pi_{\sigma}(x)\right) d t \\
& =\pi_{\sigma}\left(\int \varphi(t) \sigma_{t}(x) d t\right)=\pi_{\sigma}(\sigma(\varphi) x)
\end{aligned}
$$

for every $x$ in $A$ due to the $\sigma$-weak continuity of $\pi_{\sigma}$. Since $\pi_{\sigma}$ is faithful, we get that $\sigma(\varphi)=0$ whenever $\tilde{\sigma}(\varphi)=0$. On the other hand, suppose $\sigma(\varphi)=0$. Linear combinations of elements of the form

$$
y=u_{\sigma}\left(t_{1}\right) \pi_{\sigma}\left(x_{1}\right) u_{\sigma}\left(t_{2}\right) \pi_{\sigma}\left(x_{2}\right) \ldots \pi_{\sigma}\left(x_{n}\right) u\left(t_{n+1}\right)
$$

with $t_{i} \in G, x_{i}$ in $A, n=1,2, \ldots$ are $\sigma$-weakly dense in $R(A ; \sigma)$. Setting $z$ equal to

$$
z=\sigma_{s_{1}}\left(x_{1}\right) \sigma_{s_{2}}\left(x_{2}\right) \ldots \sigma_{s_{n}}\left(x_{n}\right),
$$

where $s_{i}=t_{1}+\ldots+t_{i}(1 \leqq i \leqq n+1)$, we may write $y$ as

$$
y=\pi_{\sigma}(z) u_{\sigma}\left(s_{n+1}\right) .
$$

Thus, we get that

$$
\begin{aligned}
\tilde{\sigma}(\varphi) y & =\int \varphi(t) \tilde{\sigma}_{t}(y) d t \\
& =\pi(\sigma(\varphi) z) u_{\sigma}\left(s_{n+1}\right)=0 .
\end{aligned}
$$

We conclude that $\tilde{\sigma}(\varphi)=0$ due to the $\sigma$-weak continuity of $\tilde{\sigma}(\varphi)$. Thus, we have shown that $\sigma(\varphi)=0$ if and only if $\tilde{\sigma}(\varphi)=0$.

Now we have that

$$
\begin{aligned}
\operatorname{Sp} \sigma & =\{\chi \in \hat{G} \mid \hat{\varphi}(\chi)=0 ; \sigma(\varphi)=0\} \\
& =\{\chi \in \hat{G} \mid \hat{\varphi}(\chi)=0 ; \tilde{\sigma}(\varphi)=0\}=\operatorname{Sp} \tilde{\sigma} .
\end{aligned}
$$

From Theorem 11 and the preceding paragraph we get

$$
\operatorname{ker} \sigma=\left\{\varphi \in L^{1}(G) \mid \hat{\varphi}(\operatorname{Sp} \sigma)=\{0\}\right\} .
$$

\section{BIBLIOGRAPHY}

1. C. Akemann and P. Ostrand, The spectrum of a derivation of $\mathrm{C}^{*}$-algebra, J. London Math. Soc. (2) 13 (1976), 525-530.

2. W. Arveson, On groups of automorphisms of operator algebras, J. Functional Analysis 15 (1974), 217-243.

3. A. Connes, Une clasification des facteurs de type III, Ann. Sci. Ecole Norm. Sup. (4) 6 (1973), 133-152.

4. J. Dixmier, Les algebres d'opérateurs dans Tespace Hilbertien, Gauthier-Villars, Paris, 1969.

5. A. Gleason, Projective topological spaces, Illinois J. Math. 2 (1958), 482-489. 
6. J. Glimm, A Stone-Weierstrass theorem for C*-algebras, Ann. of Math. (2) 72 (1960), 216-244.

7. P. Halmos, Continuous functions of hermitian operators, Proc. Amer. Math. Soc. 31 (1972), 130-132.

8. H. Halpern, Essential central spectrum and range for elements of a von Neumann algebra, Pacific J. Math. 48 (1972), 349-380.

9. E. Hille and R. Phillips, Functional Analysis and Semi-groups, Amer. Math. Soc. Colloquium Publications, Baltimore, 1957.

10. C. Kuratowski, Topology, Academic Press, New York, 1966.

11. L. Loomis, An Introduction to Abstract Harmonic Analysis, D. van Nostrand co., New York, 1953.

12. G. Lumer and M. Rosenblum, Linear operator equations, Proc. Amer. Math. Soc. 10 (1959), 32-41.

13. D. Olesen, On nurm continuity and compactness of spectrum, Math. Scand. 35 (1974), 223-236.

14. C. Richart, General Theory of Banach Algebras, D. van Nostrand co., Princeton, 1960.

15. S. Sakai, Derivations of W*-algebras, Ann. of Math. (2) 83 (1966), 273-279.

16. S. Strătilă and S. Zsidô, An algebraic reduction theory for $\mathrm{W}^{*}$-algebras, II., Rev. Roumaine Math. Pures Appl. 18 (1973), 407-465.

UNIVERSITY OF CINCINNATI - 25

CINCINNATI, OHIO 45221

U.S.A. 